\title{
LAND-BASED MOBILE LASER SCANNING SYSTEMS: A REVIEW
}

\author{
I. Puente ${ }^{\mathrm{a}}$, H. González-Jorge ${ }^{\mathrm{a}}$, P. Arias ${ }^{\mathrm{a}^{*}}$ and J. Armesto ${ }^{\mathrm{a}}$ \\ ${ }^{\text {a }}$ Close Range Remote Sensing and Photogrammetry Group, School of Mining Engineering. Campus Lagoas- \\ Marcosende, 36310, Vigo, Spain - (ipuente, higiniog, parias, julia)@uvigo.es
}

Commission V, WG V/3

KEY WORDS: Land-based Mobile Laser Scanning, LiDAR, GNSS/INS, Multi-sensor system, Road inspection, Urban Mapping

\begin{abstract}
:
Mobile mapping has been using various photogrammetric techniques for many years. In recent years, there has been an increase in the number of mobile mapping systems using laser scanners available in the market, partially because of the improvement in GNSS/INS performance for direct georeferencing. In this article, some of the most important land-based mobile laser scanning (MLS) systems are reviewed. Firstly, the main characteristics of MLS systems vs. airborne (ALS) and terrestrial laser scanning (TLS) systems are compared. Secondly, a short overview of the mobile mapping technology is also provided so that the reader can fully grasp the complexity and operation of these devices. As we put forward in this paper, a comparison of different systems is briefly carried out regarding specifications provided by the manufacturers. Focuses on the current research are also addressed with emphasis on the practical applications of these systems. Most of them have been utilized for data collection on road infrastructures or building façades. This article shows that MLS technology is nowadays well established and proven, since the demand has grown to the point that there are several systems suppliers offering their products to satisfy this particular market.
\end{abstract}

\section{INTRODUCTION}

The concept of Mobile Mapping System (MMS) dates back to late 1980 s and early 1990 s, when the first operational landbased MMS was developed by the Centre for Mapping at the Ohio State University. Their system- called GPSVan TM integrated a code-only GPS receiver, two digital CCD cameras, two colour video cameras and several dead-reckoning sensors (two gyroscopes and a distance measurement unit on each of the front wheels), where all components were mounted on a van (Goad, 1991; Novak, 1991).

Further developments on terrestrial MMS based on GPSVanincluding GIM ${ }^{\mathrm{TM}}$ and GPSVision- all used IMUs as their deadreckoning sensors (Coetsee et al., 1994; He et al., 1996). Later independent implementations of land-based MMS added dualfrequency carrier-phase differential GPS, more accurate IMUs and more sophisticated processing techniques. Examples of some other systems include VISAT ${ }^{\mathrm{TM}}$ system, designed by the University of Calgary together with GEOFIT Inc. for mobile highway mapping (Schwarz et al., 1993), KiSS ${ }^{\mathrm{TM}}$ (Hock et al., 1995) and GI-EYE ${ }^{\text {TM }}$ (Brown, 1998).

Terrestrial mobile mapping systems have been actively researched and developed over a number of years (Ellum and El-Sheimy, 2002). More recently, the mapping sensor adopted by MMS has evolved from the digital cameras to the use of laser scanning technology, often referred to as LiDAR (Light Detection and Ranging). LiDAR has been operational for surface and object reconstruction since the mid-1990s. This technology, which is based on laser range-finding measurements of the distance between the sensor and the targeted object, provides a significant increase in data points of exceptional accuracy over traditional data capture methods.

The development of laser technology together with the improvement in the performance of GNSS/INS for direct georeferencing has broadened the range of applications of these mobile mapping systems, from now on, called mobile laser scanning systems (MLS).

"Mobile LiDAR" term is widely used for a laser scanner deployed on any mobile platform such as a van, a boat, or even a $4 \times 4$ all-terrain vehicle, and often does not imply an airborne LiDAR. However, the general principles of operation are the same for airborne and terrestrial mobile LiDAR systems and the data processing workflows are very similar or almost identical in both cases.

Airborne Laser Scanning (ALS) systems use almost solely the pulse time of flight measurement principle for ranging (Riegl, 2011; Optech, 2011; Leica, 2011; TopEye, 2011; TopoSys, 2011; Fli-Map, 2011) and have been widely used for the generation of bare-earth digital elevation models (DEMs), the estimation of forest inventory attributes, and are rapidly being adopted in operational forest management.

Terrestrial Laser Scanning (TLS) systems use the same principles as ALS, however they are ground based. Locating the scanner on the ground gives some distinct advantages for capturing discrete objects from multiple angles. TLS systems exist as tripod mounted, or mobile scanning instruments and as point-scanning devices with a partial or complete hemispherical field of view or frame-scanning devices.

TLS is more useful for capturing small (relative to those captured from an aircraft) irregular objects such as buildings, earthworks and landforms such as cliff faces which can be profiled and monitored during mining.

Some reviews about mobile mapping systems have been previously done, being some examples Novak (1995), Li (1997), Tao (1998) and Grejner-Brzezinska (2001). This study tries to illustrate the latest mobile laser scanning systems existing in the market and consequently, update previous reviews on this topic. 


\section{AN OVERVIEW OF MLS TECHNOLOGY}

The development of mobile LiDAR systems is featured by the use of multiple sensors as well as integrated sensor processing methods that perform highly detailed surveys along the trajectory of the moving vehicle.

\subsection{Hardware components}

In general, the most common mobile LiDAR system configuration integrates digital frame cameras, laser scanner, GPS receiver and antenna, inertial navigation system (INS) for acceleration and orientation measurements of the moving platform and, in most cases, a wheel-mounted Distance Measuring Indicator (DMI), that provides accurate vehicle velocity updates.

The positioning sensors, namely GPS, INS and DMI, are vehicle-oriented. They are used to determine the absolute locations of the mobile mapping platform with respect to a global coordinate system, e.g., WGS-84. The mapping sensors (laser scanners, video or digital cameras, etc.) are featureoriented. They provide the positional information of objects (features) relative to the vehicle in a local coordinate system. In addition, attributes of features can be obtained from the mapping sensors. The use of multiple camera arrays to provide $360^{\circ}$ panoramic images in the horizontal plane is very common (Figure 1a), however frequently fully integrated multiple cameras (e.g. LADYBUG unit used in the IP-S2 system, Figure $1 b)$ are used.

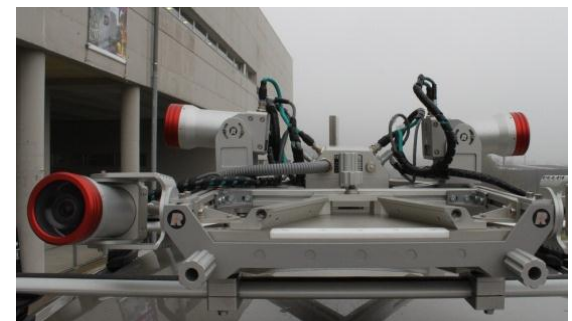

(a)

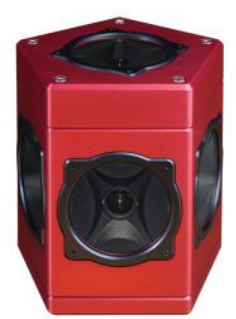

(b)
Figure 1. a) VMX-250-CS6 Camera System supporting up to six digital color cameras. b) LADYBUG with a set of six SONY CCD cameras arranged in a similar circular five-camera configuration plus a single vertical camera. (Source: Point Grey Research, 2011)

Precise calibration is required to geometrically align the positioning sensors and mapping sensors together. A sophisticated software unit controls these components and accurately synchronizes the measurements recorded through a time stamp.

A basic laser scanner measures its surroundings using LiDAR for measurements of range and angle. Currently, range measurements for mobile laser scanning systems are divided mainly into two techniques: time-of-flight and phase shift. A time-of-flight (TOF) scanner sends a short laser pulse to the target; the time that elapses between emitted pulse and received pulse correlates to the range. Examples of laser scanners using this principle include: Riegl (2011), Leica (2011), Trimble (2011), Optech (2011), 3rdTech (2011) and Sick (2011) (Figure 2a). Phase based laser scanners (Zoller+Fröhlich 2011; Faro 2011) (Figure 2b) infer the range by measuring the phase difference between the emitted and received backscattered signal of an amplitude modulated continuous wave (AM CW). Phase shift laser scanners have better accuracy, although the measurement range is lower. Highly accurate xyz-coordinates of the ground points for each laser pulse can be calculated by combining the laser range, scan angle, laser position from GPS and orientation of the laser platform from INS.

The laser pulse repetition rate (PRR) in combination with scanning mirror deflecting pattern determine LiDAR data collection rate. In the most advanced commercially available LiDAR systems, the data measurement rate is typically $50 \mathrm{kHz}$ $-500 \mathrm{kHz}$, which allows the user to collect highly accurate data of required ground point density within a very short period of time.

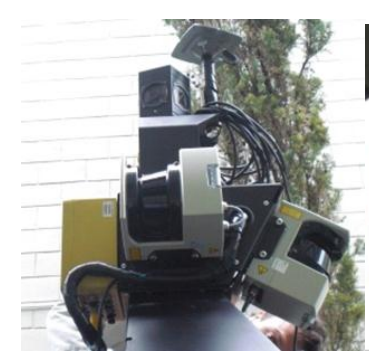

(a)

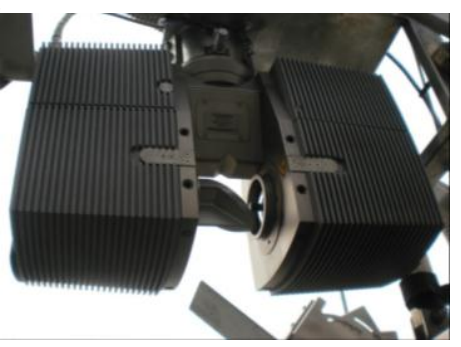

(b)
Figure 2. a) Laser SICK LMS 291 mounted on the IP-S2 system supplied by TOPCON. b) FARO PHOTON 120 mounted on ROAD-SCANNER system.

Inertial navigation provides real-time indication of position and velocity of a moving vehicle using IMU sensors that react on the basis of Newton's laws of motion.

Two primary types of IMUs are accelerometers, which sense linear acceleration in the inertial frame, and gyroscopes, which sense the inertial rotational motion (angular rates, angular increments or total angular displacements from an initial known orientation relative to inertial space). Considering an accelerometer, the first integration of acceleration gives the velocity and the second integration the position - or rather, change of position - along the accelerometer's axis.

The three types of IMUs that are most commonly used in mobile mapping systems are:(i) MEMS (Micro ElectroMechanical Systems) Gyros, that utilize tiny quartz tuning forks as sensors integrated on to sylicon chips. They are widespread used in the imaging systems that are employed on less demanding applications. Not much used in laser scanners. (ii) Fibre Optic Gyros (FOG), are very much more expensive than MEMS gyros - but gives a very acceptable performance that satisfies many laser scanning applications. Much used nowadays. (iii) Ring Laser Gyros (RLG), are the most accurate type but there are the most expensive to produce - which limits their use to high-end imaging systems and laser scanners and to only the most demanding applications in terms of accuracy.

In principle, the only external information the INS requires is initial calibration (initialization and alignment), which includes the externally provided 3D initial position, velocity and attitude. The sensor errors grow with time; therefore INS must be recalibrated periodically to maintain reliable navigation quality. In stand-alone mode, the INS navigation results are primarily affected by initial sensor misorientation, accelerometer biases and gyro drifts that cause time-dependant positioning errors.

Ground based navigation solution, integrated with the laser scanner, is a critical component for any mobile LiDAR system 
as it is used to obtain geo-referenced coordinates of the collected LiDAR data.

Data geo-referencing is achieved with an on-board position and orientation system (POS) that controls GPS and IMU data collection and processed with a Kalman filter. The GPS data helps to remove the error from the IMU drifting, while IMU data reduces the error of GPS measurements and/or maintain the navigation solution during periods of GPS outages.

A navigation solution with data of exceptional positioning accuracy is generated by a POS-MMS software tool with tightly coupled processing' that references the simultaneous process of raw GPS, IMU, DMI data and secondary GPS data, if exists.

Since the accuracy of the laser scanner instrument is on the order of few centimeters, the overall accuracy of any integrated mobile LiDAR system is often determined by the accuracy of the navigation solution.

\subsection{Data acquisition and processing}

In the data acquisition phase, three sub-systems record data as follows: the GPS/INS system calculates the trajectory and records the triggering events from the laser scanner input; the laser scanner measures two-dimensional points (i.e. profiles) and the camera system captures scenery images synchronously with the DMI displacement.

Profile data is converted into three dimensions in the georeferencing phase where each of the laser points are assigned an appropriate time stamp and coupled with the trajectory information of the scanner's location and altitude.

The necessary first step before starting the georeferencing procedure is the computation of the corrected vehicle's trajectory. For this purpose, MLS must remain totally stationary five minutes before and after the survey for INS alignment to match position with GPS and to estimate the alignment variables. Shortly afterwards, GPS data of MLS are processed by kinematic differential GPS approach using, in most cases, software packages that combine the IMU sensor using a Kalman filter. It requires a reference station that should be located on a known vertex in a barycentre position respect the underground track. In Europe, ETRS89 coordinates of this vertex, which belongs to the National Reference Network, are used for the adjustment of the survey. Additionally, mobile base station could be used to provide a surveyed point close or within the test area.

\section{MLS APPLICATIONS}

Because it employs dynamic data acquisition, mobile scanning technology can be directly used in Highway-Related applications, such as traffic sign inventory, vertical and horizontal clearance, generation of road network databases, and road surface condition inspection. There are several advantages to using mobile mapping technology in highway applications. The data acquisition is performed without blocking the traffic assuming that traffic velocity is less than, for example, $70 \mathrm{~km} / \mathrm{h}$. The information obtained is diverse - single collection can be used for multiple purposes. Moreover, since data can be both collected and processed in a short period of time, frequent and repetitive road surveys and database updating are both possible and affordable.

Objects along roads and highways, for example road size, traffic signs, road centerlines, posters, light poles, potholes,pavements, fences, sidewalks, guard-rails, bridges, tunnels, retaining walls and overhead wires, are usually represented as clear features in the scanning and image sequences. Therefore, they can be identified easily and measured interactively to build facility management databases.

It is often required in the inspection of road surface condition that road surface cracks are located and measured. Current MLS systems are not able to perform crack surveys to the required level of detail for asphalt maintenance, but being the system equipped with laser sensors (normally two), the depths between the sensors and the road surface are available as relative measurements. If the control data from GPS and INS are available, the depth data derived from the laser sensors can be integrated into the global reference system and used to generate a Digital Road Surface Model (DRSM). The DRSM provides a geometric description of the road surface. The use of two LiDAR heads increases the achievable resolution. It offers the flexibility of collecting data at a higher resolution at the same vehicle speed, or maintaining the same resolution but collecting the data at higher vehicle speeds.

Also, the end user could enjoy these other final products:

1. 3D-routes and topologically connected road graphs, compatible with the most common Geographical Information Systems.

2. Geo-referenced images acquired with the video system in JPEG or AVI format, used in the post-processing phase, are also delivered to the end user for virtual inspection of the road network. Virtual inspections through the consultations of movie are highly regarded by the public administration because they allow a quick and easy check of the status of the road network.

3. Road cross sections surveyed with a laser-scanner. The resulting sections have an angular and a distance range at a certain step which is variable by vehicle speed and laser used. The clouds of scan-points are geo-referenced.

4. Digital Terrain Models, especially for rapid volume determination.

5. 3D city models with detailed façade and street level information.

\section{EXISTING MLS IN CURRENT MARKET}

MLS suppliers include several large companies such as TOPCON and TRIMBLE and medium size companies as OPTECH, RIEGL, MDL or SITECO which are already wellestablished as suppliers of surveying instrumentation and laser scanning systems for mapping industry.

\subsection{ROAD-SCANNER - SITECO}

Since 2005, in collaboration with the universities of Parma and Bologna, SITECO has carried out a research on mobile mapping giving as a result the ROAD-SCANNER system. The vehicle integrates a LANDINS navigation system supplied by IXSEA, based on fiber-optic technology; a set of 8 lastgeneration BASLER SCOUT cameras, where two cameras are located in front of the vehicle to form a perfect stereo couple, one on the right and one on the left side. Four more cameras are mounted with a slant of $45^{\circ}-135^{\circ}-225^{\circ}-315^{\circ}$ respectively, to assure a full vision of the road. Moreover, it includes an helical scanning through the FARO PHOTON 120. The ROADSCANNER system aims to provide a complete road inspection 
unit. For this purpose, it integrates a profilometer sensor from DYNATEST for computing longitudinal roughness and IRI and a ground penetrating radar from IDL for pavement layers inspection (Siteco, 2011).

\subsection{IP-S2 - TOPCON}

TOPCON Positioning Systems has introduced its mobile mapping system, the IP-S2, in the spring of 2009. It includes a TOPCON dual-frequency 40-channel GNSS receiver operating at $20 \mathrm{~Hz}$, which is coupled to a Honeywell HG1700 tacticalgrade IMU based on a ring laser gyro at $100 \mathrm{~Hz}$ operation. The resulting GPS/IMU positional data is supplemented by that generated by a wheel-mounted DMI with an angular encoder operating at $30 \mathrm{~Hz}$ to complete the overall positioning capability. Besides these positioning devices, the imaging capability is based on the LADYBUG multicamera unit that carries out the $360^{\circ}$ panoramic imaging with framing rates up to 15 frames per second. The IP-S2 uses three SICK LMS 291 scanners operating at $75 \mathrm{~Hz}$. Currently, Topcon has almost completely substituted their SICK lasers for an integrated VELODYNE HDL-64E S2 High definition LiDAR, consisting of a battery of 64 lasers and providing 3D data at a rate of 1.3 million points per second (Topcon, 2011).

\subsection{MX8 - TRIMBLE}

The TRIMBLE MX8 Mobile Spatial Imaging System is an advanced mobile data capture system that combines imaging and laser scanning capabilities to measure objects. It was available in the third quarter of 2010 through Trimble's GeoSpatial sales channels.

Featuring a pod-type design (Figure 3a), it is easily redeployed and installed on a variety of vehicles as project demands change. The data capture system integrates a GPS/IMU subsystem used for geo-positioning, namely, the POS LV 420 supplied by APPLANIX (another TRIMBLE company), including two TRIMBLE GPS receivers (for accuracy detection of the vehicle heading). The system integrates two RIEGL VQ250 scanners and a panoramic imaging system composed up to six 5MP digital cameras. Another 5MP camera oriented for surface imaging completes the system.

Besides the hardware aspects of the overall system, Trimble have also developed a series of software packages. These include the TRIMBLE Trident-3D Data Capture Software for data acquisition and TRIMBLE-3D Analyst for data extraction and processing, including the semi-automatic detection, recognition and extraction of signs, poles, lanes or vertical clearances among others (Trimble, 2011).

\subsection{STREETMAPPER Portable - 3D LASER MAPPING Ltd\& IGI mbH}

3D LASER MAPPING Ltd. has acted as a system integrator in developing its portable STREETMAPER system, in close collaboration together with the German systems supplier, IGI $\mathrm{mbH}$. The innovative design of STREETMAPPER Portable offers the same top-specification sensors as the STREETMAPPER 360 system, but built around a lightweight mounting system and compact control and power unit. It is a mobile, LiDAR-based terrain mapping system consisting of: one 2D LiDAR sensor (RIEGL VQ-250) with $300 \mathrm{kHz}$ measurement rate, $200 \mathrm{~m}$ range and unlimited returns per pulse; GPS and FOG-based IMU components combined in the TERRAcontrol navigation system and a Camera system comprising one digital 12Mpixel SLR (single-lens reflex) camera with timer unit for precise positioning of each image (3D laser mapping Ltd., 2011).

\subsection{VMX-250 - RIEGL}

The new RIEGL VMX-250 is an extremely compact and userfriendly Mobile Laser Scanning (MLS) measurement unit. The roof-carrier mounted measuring head (Figure $3 b$ ) integrates two RIEGL VQ-250 scanners, inertial and satellite navigation hardware, and mounting points for digital cameras or video equipment. Each VQ-250 provides a "full circle" $360^{\circ}$ scan within its 2D scanning plane and can measure ranges of $200 \mathrm{~m}$ (with $80 \%$ reflectivity) with PRR ( Pulse Repetition Rate) values up to $300 \mathrm{kHz}$ and line scan speeds up to 100 lines per second, while maintaining an accuracy of 10mm (Riegl, 2011).

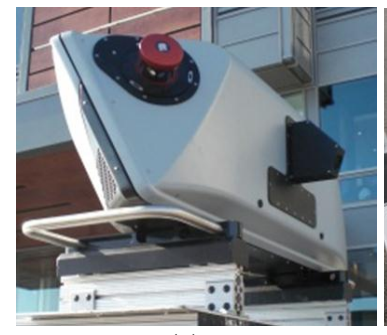

(a)

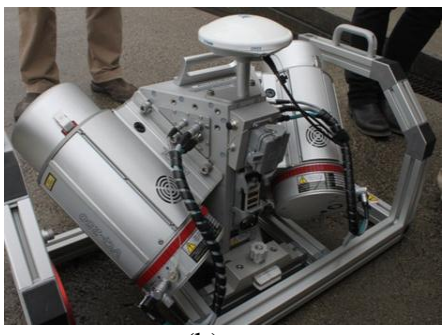

(b)
Figure 3. a) MX8 data camera system from TRIMBLE. b) RIEGL VMX-250 measuring head.

\subsection{DYNASCAN - MDL Laser Systems}

MDL Laser Systems presented its new mobile mapping scanner DYNASCAN at the SPAR conference 2010 in Amsterdam. The DYNASCAN 'plug and play' LiDAR system contains a seamlessly integrated INS, RTK GNSS system and high speed laser scanner all in one pod.

The system is lightweight, small-size highly portable and may be mounted on vehicles or vessels to acquire 3D data of objects in a range up to $500 \mathrm{~m}$.

There are two versions of DYNASCAN, one with a single MDL Scanning laser module (Figure 4a) and another one, dual. Those operate up to $30 \mathrm{~Hz}$ with PRR values of $36 \mathrm{kHz}$, while maintaining an accuracy of $5 \mathrm{~cm}$.

A DYNSCAN system comes complete with data acquisition and post processing software suite. Data from all available sensors is synchronised, "time tagged" and recorded. Post processed and raw data may be exported to most $3 \mathrm{D}$ data base and CAD software packages (MDL Laser Systems, 2011).

\subsection{LYNX Mobile Mapper - OPTECH}

The LYNX Mobile Mapper was released towards the end of 2007 by OPTECH, generating rich survey-grade LiDAR and image data at highway speeds. LYNX scanners (Figure 4b) have a maximum range of $200 \mathrm{~m}$; a full circle $360^{\circ}$ angular coverage; a pulse measurement rate up to $500 \mathrm{kHz}$ and a scan rate of 100 Hz. The positioning system was designed by APPLANIX (POS LV 420) and the GNSS receivers belong to TRIMBLE. The system control software enables you to select the camera image frame size for highly efficient image capture. The result: extremely dense data in a smaller project deliverable. The LYNX Mobile Mapper delivers project data with the highest accuracies commercially available (Optech, 2011). 


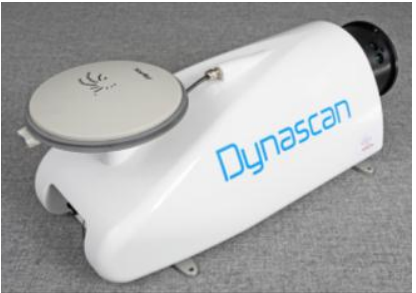

(a)

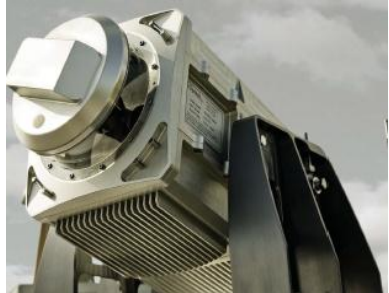

(b)
Figure 4. a) Single Laser DYNASCAN Pod. (Source: $M D L$ Laser Systems, 2011) b) The OPTECH LYNX laser scanner that generates a 2D profile scanning pattern. (Source: Optech, 2011)
There are other suppliers of MLS systems such as MITSUBISHI ELECTRIC CORPORATION or GOOGLE Inc. MMS type S, MMS type X (640, 440 and 220) or 'The Google Car', which is responsible for creating the GOOGLE STREET VIEW images, are some examples of other MLS systems. GOOGLE STREET VIEW vehicles use IP-S2 system supplied by TOPCON; however, LADYBUG multiple cameras have been replaced by the now multiple camera system, that comprises nine individual CCD cameras, eight of which are arranged, spaced equally, in a concentric ring pointing outwards, with the ninth camera pointing vertically upwards. Further information about these systems can be found in Petri, G. (2010).

\begin{tabular}{|c|c|c|c|c|c|c|c|}
\hline $\begin{array}{l}\text { MLS } \\
\text { System }\end{array}$ & Road Scanner & IP-S2 Compact & MX8 & $\begin{array}{l}\text { StreetMapper } 360 \\
\text { or Portable }\end{array}$ & VMX-250 & Dynascan & Lynx Mobile Mapper \\
\hline Scanner & Faro Photon 120 & Sick LMS 291 & \multicolumn{3}{|c|}{ VQ-250 } & MDL scanner & Lynx laser scanner \\
\hline $\begin{array}{l}\text { Maximun } \\
\text { range }\end{array}$ & $120 \mathrm{~m}(\rho 90 \%)$ & $80 \mathrm{~m}(\rho 10 \%)$ & \multicolumn{3}{|c|}{$200 \mathrm{~m}(\rho 80 \%)$} & up to $500 \mathrm{~m}$ & $200 \mathrm{~m}(\rho 80 \%)$ \\
\hline $\begin{array}{l}\text { Range } \\
\text { precision }\end{array}$ & $\begin{array}{l}1 \mathrm{~mm} @ \\
25 \mathrm{~m}, \rho 90 \%\end{array}$ & 10 mm @20m & \multicolumn{3}{|c|}{$5 \mathrm{~mm} @ 150 \mathrm{~m},(1 \sigma)$} & & $8 \mathrm{~mm}, 1 \sigma$ \\
\hline $\begin{array}{l}\text { Range } \\
\text { accuracy }\end{array}$ & $\pm 2 \mathrm{~mm} @ 25 \mathrm{~m}$ & $\pm 35 \mathrm{~mm}$ & \multicolumn{3}{|c|}{ 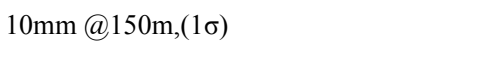 } & $\pm 5 \mathrm{~cm}$ & $\pm 10 \mathrm{~mm},(1 \sigma)$ \\
\hline PRR & $122-976 \mathrm{kHz}$ & $40 \mathrm{kHz}$ & \multicolumn{3}{|c|}{ up to $600 \mathrm{kHz}(2 \times 300 \mathrm{kHz})$} & $36 \mathrm{kHz}$ & $\begin{array}{l}1000 \mathrm{kHz}(2 \times 500 \\
\mathrm{kHz})\end{array}$ \\
\hline $\begin{array}{l}\text { Scan } \\
\text { speed }\end{array}$ & $48 \mathrm{~Hz}$ & $75 \mathrm{~Hz}$ & \multicolumn{3}{|c|}{$200 \mathrm{~Hz}(2 \mathrm{x} 100 \mathrm{~Hz})$} & up to $30 \mathrm{~Hz}$ & $200 \mathrm{~Hz}(2 x 100 \mathrm{~Hz})$ \\
\hline $\begin{array}{l}\text { Scanner } \\
\text { FOV }\end{array}$ & $\mathrm{H} 360^{\circ} / \mathrm{V} 320^{\circ}$ & $180^{\circ(1)} / 90^{\circ(2)}$ & \multicolumn{3}{|c|}{$360^{\circ}$ without gaps } & $360^{\circ}$ & $360^{\circ}$ without gaps \\
\hline $\begin{array}{l}\text { Angular } \\
\text { resolution }\end{array}$ & $\begin{array}{l}\mathrm{H} 0,00076^{\circ} / \\
\mathrm{V} 0,009^{\circ}\end{array}$ & $1^{\circ(1)} / 0,5^{o(2)}$ & \multicolumn{3}{|c|}{$0,001^{\circ}$} & $0,01^{\circ}$ & $0,001^{\circ}$ \\
\hline Weight & $14.5 \mathrm{~kg}$ & $22.7 \mathrm{~kg}$ & \multicolumn{3}{|c|}{ approx. $11 \mathrm{~kg}$} & $11 \mathrm{~kg}$ & $78 \mathrm{~kg}$ \\
\hline
\end{tabular}

(1) Sick LMS 291-S05 used in IP-S2 Compact system.

(2) Sick LMS 291-S14 used in IP-S2 Compact system.

Table 1. Laser scanner specifications of MLS systems reviewed.

The ROAD-SCANNER system uses a non-showerproof 3D Faro Photon 120, a phase difference scanner showing intermediate solutions between TOF scanners (mainly used for long-range measurement applications) and high-accurate triangulation ones. Although it is a $3 \mathrm{D}$ scanner, it works as a $2 \mathrm{D}$ device with its horizontal movement fixed when it is mounted on the vehicle. Because of its physical configuration, it has a blind spot (vertical FOV is $320^{\circ}$ ) that SITECO typically locates in the zenith.

TOPCON IP-S2 uses three SICK LMS 291 scanners (Sick, 2011) that measure a short range $(80 \mathrm{~m}$ maximum under field conditions of $\rho 10 \%$ ) with low accuracy values around $35 \mathrm{~mm}$, scan speed of 75 lines per second and a low measurement rate of 40000 points per second. This system has been proven to provide panoramic views along many streets in the world but it seems not to be the best option for a survey/Inspection activity, where more accurate and detailed point data is required. The same inconvenience happens to the MDL scanner from DYNASCAN because, although it enables to undertake detailed 3D large scale mapping projects, measuring long ranges up to $500 \mathrm{~m}$, pulse repetition rate and scan speed parameters are lower in comparison with the rest of MLS systems using RIEGL VQ-250 or with the one from OPTECH.

The latter shows the best laser specifications existing in the market, collecting quite precise XYZ data at a rate of 1,000,000 points per second (for two sensors).
Each sensor detects and records up to four distinct return measurements for each outgoing laser pulse, including the last return. This feature is only superseded by the VQ-250, that using an online waveform processing and echo digitization technology, registers practically an unlimited number of targets per pulse.

\section{CONCLUSIONS}

In this work, a new review on land-based mobile laser scanning (MLS) to derive detailed topographical data was described. Mainly it could be distinguish some systems that are being used for cartographic mapping applications such as the TOPCON IPS2 system or "The Google Car" versus those ones collecting data about the infrastructures (road, rails, bridges, tunnels...) that are needed for inspection, engineering and management purposes. The accuracy requirements for the map or survey data change considerably, so every scanner specification should be taken into account in order to obtain the best solution according to its aim.

Within this paper, the development of mobile laser scanning systems since the very beginning is detailed, together with a basic hardware components overview and also, the main applications in the current market. However it should be noted that there are other mobile mapping systems being operated individually by their companies that were not included in this review.

Is a fact that MLS systems have demonstrated their power in wide application areas and are gaining widespread acceptance in the course of time. With the continuous advancement of mobile mapping technology as well as the reduced costs of the 
system development, mobile mapping potential will be explored further.

\section{References}

3D laser mapping Ltd., 2011. Homepage of the company 3D laser mapping Ltd.

http://www.3dlasermapping.com (accessed March 2011)

3rdTech, 2007.Homepage of the company 3rdTech.

http://www.3rdtech.com (accessed March 2011)

Brown, A., 1998. "High Accuracy Targeting Using a GPSAided Inertial Measurement Unit". In Proceedings of the $54^{\text {th }}$ Annual Meeting. The Institute of Navigation (ION), Denver, CO.

Coetsee, J., Brown, A. and Bossler, J., 1994. "GIS Data Collection Using The Gpsvan Supported By A GPS/Inertial Mapping System". In Proceedings of GPS-94. The Institute of Navigation (ION), Salt Lake City, UT.

Ellum, C. and El-Sheimy, N., 2002. "Land-Based Integrated Systems for Mapping and GIS Applications”. Survey Review, 36(283).

Faro, 2007.Homepage of the company FARO Technologies,Inc. http://www.faro.com (accessed March 2011)

Fli-Map, 2011. Homepage of FLI-MAP.

http://www.flimap.nl (accessed March 2011)

Goad, C. C., 1991. "The Ohio State University Mapping System: The Positioning Component". In Proceedings of the $47^{\text {th }}$ Annual Meeting, pp.121-124. The Institute of Navigation (ION), Williamsburg, VA.

Grejner-Brzezinska, D. A., 2001. "Mobile Mapping Technology: Ten years later ( Part 1)". Surveying and Land Information Systems ( SaLIS), 61(2): p.75.

He, G., Orvets, G. and Hammersley, R., 1996. "Capturing Urban Infrastructure Data Using Mobile Mapping System”. In Proceeding of the 52 nd Annual Meeting, pp. 667-674. The Institute of Navigation (ION), Cambridge, MA.

Hock, C., Caspary, W., Heister, H., Klemm, J. and Sternberg, H., 1995. "Architecture and Design of the Kinematic Survey System KiSS". In Proceeding of the $3^{\text {rd }}$ International Workshop on High Precision Navigation, pp. 569-576. Stuttgart, Germany.

Leica, 2007. Homepage of the company Leica Geosystems. http://ww.leica-geosystems.com (accessed March 2011)

Li, R., 1997. "Mobile Mapping: An Emerging Technology for Spatial Data Acquisition". Photogrammetric Engineering and Remote Sensing (PE\&RS), 63(9):pp. 1085-1092.

MDL Laser Systems, 2011 . Homepage of the company MDL Laser Systems.

http://www.mdl.co.uk (accessed March 2011)

Novak, K., 1991. "The Ohio State University Mapping System: The Stereo Vision System Component". In Proceedings of the $47^{\text {th }}$ Annual Meeting, pp.121-124. The Institute of Navigation (ION), Williamsburg, VA.

Novak, K., 1995. "Mobile Mapping Technology for GIS Data Collection". Photogrammetric Engineering and Remote Sensing (PE\&RS), 61(5):pp. 493-501.

Optech, 2011. Homepage of the company Optech. http://www.optech.ca (accessed March 2011)

Petri, G., 2010. "An Introduction to the Technology Mobile MappingSystems”.GeoInformatics 13(1), pp. 32-43.

Point Grey Research, 2011. Homepage of the company Point Grey Research.

http://www.ptgrey.com (accessed March 2011)

Riegl, 2011. Homepage of the company RIEGL Laser Measurement Systems GmbH.

http://www.riegl.com (accessed March 2011)

Schwarz, K.-P., Martell, H., El-Sheimy, N., Li, R., Chapman, M. and Cosandier, D., 1993. "VISAT- A Mobile Highway Survey System of High Accuracy". In Proceedings of the Vehicle Navigation and Information Systems Conference, pp. 467-481. Institute of Electrical and Electronics Engineers (IEEE), Ottawa, Canada.

Sick, 2011. Homepage of the company Sick Group. http://www.sick.com (accessed March 2011)

Siteco , 2011. Homepage of the company Siteco Informatica. http://www.sitecoinf.it (accessed March 2011)

Tao, C. V., 1998. “ Towards Sensor Integrated Technology to Fast Spatial Data Acquisition" In. Proc. of Int'l Workshop on Urban Multi-Medial 3s Mapping, 08-09 June, Tokyo, Japan, pp. 97-104.

Topcon, 2011. Homepage of Topcon Global Gateway. http://www.topconpositioning.com (accessed March 2011)

TopEye, 2011. Homepage of the BLOM GROUP. www.blomasa.com (accessed March 2011)

TopoSys, 2011. Homepage of the company TopoSys GmbH. http://www.toposys.com (accessed March 2011)

Trimble, 2011. Homepage of the company Trimble. http://www.trimble.com (accessed March 2011)

Ussyshkin, V., 2009. "Mobile Laser Scanning Technology for Surveying Application: From Data Collection to End-Products". FIG Working Week 2009Surveyors Key Role in Accelerated Development, Israel, 3-8 May 2009

Zoller+Fr"ohlich, 2011. Homepage of the company Zoller+Fr"ohlich GmbH.

http://www.zofre.de (accessed March 2011)

\section{Acknowledgements}

The authors want to thank Xunta de Galicia and Ministry of Science and Innovation of Spain for the financial support (Project code BIA2009-08012, human resources programs IPP055 and BES-2010-034106). 